\title{
La constitución de lo sensible en la fenomenología de Edmund Husserl: Acerca de la relación entre la síntesis temporal y la asociación
}

\author{
The constitution of the sensible in Edmund \\ Husserl's phenomenology: \\ Approaching the relation \\ between temporal synthesis and association
}

\author{
VERÓNICA KRETSCHEL \\ UBA-CONICET-CEF
}

Recibido:18/06/15 Aceptado:07/09/15

\section{RESUMEN}

La fenomenología genética procura poner de manifiesto las condiciones según las cuales se constituye el material sensible en la inmanencia de la conciencia. Se determina así un ámbito de la vida yoica que ocurre en la antesala del yo. La síntesis temporal se resignifica, en los estudios sobre la génesis, en tanto primera dimensión pasiva de la conciencia. Nuestro objetivo es, aquí, establecer de qué modo se relaciona esta síntesis con los procesos pasivos de asociación.

PALABRAS CLAVE:

FENOMENOLOGÍA-HUSSERL-PASIVIDAD-ASOCIACIÓN

CONCIENCIA TEMPORAL

\footnotetext{
ABSTRACT

Genetic phenomenology aims to expose the conditions for the constitution of the sensible material in the inmanence of the consciousness. Thus, a field of the ego-life is determined as the «front room of the ego». From a genetic point of view, temporal synthesis is resignified as the 
first passive dimension of the consciousness. Our goal here is to establish how this synthesis is related to the passive association process.

KEYWORDS:

PHENOMENOLOGY-HUSSSERL-PASSIVITY-ASSOCIATION

TEMPORAL CONSCIOUSNESS

Tanto Para el EMPIRISMo de David Hume, como para la filosofía trascendental kantiana la sensación es algo dado. La necesidad de que lo sensible inhiera en el sujeto representa el comienzo de la actividad cognoscitiva. La fenomenología husserliana, por su parte, reconoce esta necesidad, pero plantea, a la vez, la pregunta acerca de la constitución de la sensación. Puede establecerse un recorrido en la filosofía de Husserl cuyo punto de partida radica en describir a la sensación como algo neutro, al proponer que la constitución del objeto depende de la capacidad interpretativa de los actos noéticos (a principios del s. XX en las Investigaciones Lógicas, por ejemplo), y que se vuelve hacia ' 1920 en torno a la constitución pasiva del material sensible en la inmanencia de la conciencia. Creemos que estos análisis pueden leerse como una radicalización de los límites que encuentra el modelo de las Investigaciones Lógicas en el estudio de la conciencia temporal. Esto es: si el modelo aprehensión-contenido de aprehensión fracasa a la hora de dar cuenta de los actos temporales, en la medida en que se pone en evidencia el carácter siempre presente de la sensación, la voluntad de establecer los procesos que operan en la conformación del dato hylético terminan por fundar todo acto en una dimensión pasiva de la vida de la conciencia. Mientras que en el primer caso la sensación restringía la potencia de la interpretación solo en relación con el tiempo, se afirma en el último caso una vida de lo material independiente de toda actividad yoica como parte de la subjetividad. Nos proponemos aquí ahondar en este camino recorrido por la fenomenología de Husserl y, puntualmente, determinar cuál es la relación que puede establecerse entre la síntesis del tiempo y los procesos asociativos, en tanto modos de organización del material sensible. En otras palabras, pretendemos responder a la pregunta por el lugar que ocupa la conciencia del tiempo cuando la fenomenología destaca la importancia de la constitución pasiva del dato hylético. 


\section{GÉNESIS, ASOCIACIÓN Y PASIVIDAD}

«Génesis, asociación y pasividad son para Husserl tres títulos de problemas que se continúan $»^{1}$ señala Elmar Holenstein. La dirección genética de la fenomenología hace referencia a una dimensión pasiva de la vida de la conciencia y a la síntesis de asociación como un modo de organización de la experiencia característico de dicha esfera. Es así, en la medida en que es la fenomenología genética la que posibilita una apropiación fenomenológica de las dinámicas asociativas; esto es: el nuevo enfoque amplía el campo de la investigación en dirección a una esfera en la que operan procesos pasivos de asociación. Aunque pueden encontrarse menciones a tópicos relacionados ya en la fenomenología temprana, recién en los años '20 se lleva a cabo un estudio sistemático de sus procesos. A su vez, los desarrollos en torno a su operar conducen a una investigación general acerca de la pasividad. La síntesis temporal es resignificada en este contexto como estrato primero de la experiencia que ocurre sin intervención activa del sujeto. Buscando establecer cuáles son las relaciones que se concretan entre temporalidad y asociación, desarrollaremos los distintos niveles en qué operan las síntesis pasivas de asociación.

Un modo de acceder a la temática de la asociación es a través de la noción de sensación. En el marco del esquema aprehensión-contenido de aprehensión, ${ }^{2}$ Husserl asume la neutralidad de los contenidos y recarga todo el peso en la conformación de los correlatos intencionales en el aspecto aprehensivo de la relación. Es decir, que es la interpretación de los contenidos la que establecería la referencia objetiva. Junto con esto hay que señalar que es, justamente, la tesis acerca del carácter neutro de la sensación lo que pone en jaque el esquema. En textos complementarios a las Lecciones de fenomenología de la conciencia interna del tiempo se comienza a plantear la posibilidad de atribuirle a un contenido cualquier determinación temporal, para, finalmente, negar esta opción. En este contexto, podemos pensar que la asociación viene a explicar cómo se constituyen los contenidos y en qué medida operan en la articulación de una referencia objetiva. Tengamos en cuenta que, mientras que la aprehensión se relaciona con una operación activida del yo, las síntesis de asociación representan procesos que ocurren pasivamente $\mathrm{o}$, en otras palabras, sin intervención yoica.

1 Holenstein 1972, 26.

2 El esquema aprehensión-contenido de aprehensión (SchemaAuffassung-Auffassungsinhalt) es el modelo empleado por Husserl para dar cuenta de la constitución de los actos en las Investigaciones Lógicas. Es puesto en duda en el marco del análisis de la constitución de la conciencia temporal en los textos tempranos sobre el tiempo (Husserl 1969) y restringido a la constitución de la percepción. Para una primera lectura, ver. Brough 1977, donde se desarrollan los aspectos que conducen a Husserl al descubrimiento de la conciencia absoluta constituyente del tiempo y la consiguiente restricción del esquema. 
Esta indicación nos permite relacionar la problemática con otra cuestión abierta en las Lecciones. Se suele afirmar que en estos textos Husserl prioriza la fase protoimpresional de la conciencia constituyente del tiempo. ${ }^{3}$ Esta prioridad puede entenderse en términos de su función en tanto instancia de donación de lo ajeno a la conciencia. La protoimpresión era considerada allí, en primer lugar, como una fase receptiva. ${ }^{4}$ Esta mera recepción se transforma, posteriormente, en un proceso sintético. En el presente la conciencia no sólo recibe a lo otro de sí, sino que lo organiza a través de operaciones que motivan la atención del yo para la constitución de un objeto trascendente. La protoimpresión como un proceso de autotemporalización originario -la genesis spontanea del Apéndice I de las Lecciones- es un antecendente fuerte de la idea de constitución pasiva. En este sentido, afirma Bruce Bégout:

Aquello que es decisivo en este cuestionamiento, por parte de Husserl, del esquema central de la aprehensión intencional, pensada como animación de una materia inerte por actos formales, es, precisamente, el hecho de que la sensibilidad no es más relegada al grado de la receptividad inactiva y cuasi muerta, sino que ella posee la facultad de preconstituir por sí misma las unidades temporales en el sentir del tiempo y de la duración fluyente. ${ }^{5}$

Es así que los análisis sobre la organización de los contenidos en el presente viviente (que veremos bajo el concepto de protoasociación) pueden leerse como una ampliación de la idea aquí señalada de constitución. Mientras que en los textos de las Lecciones encontramos un proceso pasivo de autotemporalización que se establece desde la protoimpresión en el presente, en los Análisis se presentan, dentro de los límites de esa instancia de la conciencia, los mecanismos que conforman pasivamente las unidades hyléticas que habilitan al sujeto para la constitución del objeto intencional. Creemos que esta posibilidad de leer las Lecciones como antecedente de los Análisis permite sostener que la síntesis temporal representa el estrato primero de la dimensión pasiva de la conciencia.

Las modificaciones que se producen en la noción de sensación implican cambios en la concepción de sujeto. Esta dimensión pasiva de la vida de la conciencia refiere a operaciones que trascienden las posibilidades de un sujeto concebido como centro de la actividad constituyente. Un sujeto pasivo subyace

3 Este tópico nos enfrenta a la discusión acerca de la pertenencia o no del pensamiento de Husserl a la metafísica de la presencia. Sobre esta discusión ver, entre otros: Bernet 1982, Bernet 1983 Derrida 1995, Brough 1993. O la reconstrucción que he realizado sobre este tema en Kretschel 2013.

4 Acerca de la pasividad entendida como pasividad en la fenomenología pre-genética, ver Holenstein, op. cit., 208-210

5 Bégout 2000, 26

Contrastes vol. XXI-N² (2016) 
a toda actividad yoica y, a su vez, en la medida en que la pasividad explica también cómo es posible que un yo tenga una historia -esto es: que devenga un sujeto concreto-, la noción de sujeto deberá incluir, entonces, una descripción de la subjetividad entendida como mónada, como sustrato de habitualidades. Ahora bien, entre el sujeto y los contenidos que se conforman asociativamente hay otra cuestión que necesitamos analizar. Decíamos que el material sensible se organiza y motiva que el yo se vuelva sobre las unidades constituidas pasivamente. Entre ese volverse del yo y las síntesis de asociación se encuentra el fenómeno de la afección, como aquello que explica las condiciones según las cuales el yo es llamado por lo sensible.

Dado este marco, nos dedicaremos en lo siguiente a desarrollar los fenómenos que implica la fenomenología de la asociación. En primer lugar, analizaremos las operaciones sintéticas que la conforman y, luego, abordaremos la noción de afección, como lo que vincula al yo con las unidades constituidas pasivamente. Por motivos de espacio, no nos referiremos en esta ocasión a las modificaciones que el descubrimiento de una dimensión pasiva de la experiencia impone a la noción de sujeto. Un estudio pormenorizado de este tema se impone para un desarrollo posterior.

\section{LA ASOCIACIÓN DESDE UN PUNTO DE VISTA GENÉTICO}

La fenomenología genética posibilita un acceso a la noción de asociación desde una perspectiva que supera aquella de la psicología asociacionista moderna. ${ }^{6}$ Es pertinente en este caso recordar las críticas de Husserl tanto al psicologismo como a Brentano. Por un lado, las asunciones metafísicas implicadas en concebir que entre el mundo y el sujeto se establecen relaciones de causa-efecto. Por otro, las consideraciones husserlianas en torno al carácter fantasioso de la asociación originaria de Brentano. La reducción es el paso metódico que permite a la fenomenología salvar estos problemas y apropiarse, si bien tardíamente, ${ }^{7}$ de la noción de asociación. Es así que en el campo de inmanencia que habilita la epojé encontramos que entre las dinámicas pasivas que operan en la conciencia ocurren síntesis pasivas de asociación. Estos procesos no son «una forma de la causalidad psicofísica objetiva, ni el modo en que el aparecer de las reproducciones, de las rememoraciones, está determinado causalmente en la vida anímica humana y animal» $:^{8}$ tanto las realidades objetivas como las causalidades objetivas han quedado puestas «entre paréntesis» por la reducción. A su vez, tampoco hablamos aquí de procesos irreales como aquellos

6 Acerca de la relación entre las nociones modernas de asociación y la de Husserl, ver, Ibid., 117 y ss.

7 Hussserl, 1973, 114. Se recoge la traducción de José Gaos en Husserl, 1996, 137

8 Husserl 1966, 117 
señalados en torno a la proteroaesthesis brentaniana. Sino que, restringidos al estudio de fenómenos intencionales, se advierte que en el presente fluente de la conciencia interviene el pasado.

«Lo presente recuerda lo pasado» ${ }^{9}$ afirma Husserl y, por ello, la conciencia perceptiva se define, también, como una conciencia evocadora. Para dar cuenta de los procesos que operan en la conformación de este modo de concebir la conciencia debemos estudiar la asociación reproductiva; es decir: llevar a cabo una teoría de la génesis de las rememoraciones efectivas y posibles. Pero, en la medida en que el presente no se asocia solamente con el pasado, sino que se dan en él también anticipaciones del futuro, aquella debe complementarse con una teoría de la génesis de las esperas y de las apercepciones vinculadas. Agrupamos los mecanismos allí puestos en juego bajo el concepto de asociación inductiva, puesto que la anticipación se efectúa en términos de un predelineamiento de la experiencia por venir en función de lo ya vivido y la inducción es el método según el cual se proyecta un futuro probable sobre la base empírica de lo ya dado. Finalmente, debemos mencionar un nivel de la asociación que se establece únicamente en el presente. Si limitamos el análisis al presente sin intervención de las fases pasadas y futuras de la conciencia, encontramos la protoasociación. Intervienen allí las leyes que dan cuenta de la constitución de las formaciones de unidad preobjetivas.

Antes de entrar en la descripción de los tres niveles de la asociación pasiva, debemos referirnos a la pregunta que conduce este trabajo y que, incluso, aparece de manera bastante obvia en el párrafo anterior.

\section{1. ASOCIACIÓN Y TIEMPO}

Parece fácil advertir que una estrecha relación une el tiempo y la asociación. Pero, ¿en qué consiste tal relación? Por una parte, Husserl sostiene una prioridad de la síntesis del tiempo sobre la asociación: «No tenemos necesidad de buscar el comienzo primordial. Evidentemente está presupuesta la síntesis que se opera continuamente en la conciencia originaria del tiempo»». ${ }^{10}$ Por otra, destaca la necesidad de estudiar los procesos asociativos tanto para dar cuenta de la vida del yo, como para entender la constitución de los objetos. Esta necesidad está motivada por el carácter abstracto de la síntesis del tiempo, entendida como «un marco formal universal, en una forma sintéticamente constituida, de la que deben participar todas las demás síntesis posibles». ${ }^{11} \mathrm{Y}$, pareciera, que es, en efecto, esta formalidad lo que conduce a la abstracción: «[la síntesis del tiempo] no es más que la conciencia que establece una forma general. La mera forma

9 Ibid., 118

10 Ibid., 125

11 Ibid., 125

Contrastes vol. XXI-N² (2016) 
es sin duda una abstracción, y del mismo modo el análisis intencional de la conciencia del tiempo y su operación es de antemano un análisis abstracto». ${ }^{12}$

El estudio de la conciencia del tiempo es formal porque lleva a cabo una abstracción: no considera el contenido. Sin embargo, cuando concebimos un objeto que dura y pensamos en qué es lo que dura, notamos que aquello que dura es el contenido. Respecto a esto, sostiene Bégout:

Que la objetividad sensible pueda, entonces, perdurar en el tiempo como una unidad de configuración fija, como una cosa «especificable», es, primero, debido a las leyes de la constitución pasiva. De aquí que toda objetividad, sensible o categorial, sea portadora de un núcleo insuprimible de sentido y que ya en la esfera pasiva se elabore una unidad de recubrimiento pasivo que se da a la atención del yo como objetividad idéntica y permanente. ${ }^{13}$

El estudio del tiempo deja por fuera un aspecto que es esencial para comprender la conformación de la identidad objetiva. Sobre todo teniendo en cuenta que los contenidos también son constituidos en la inmanencia. El análisis de la asociación, en la medida en que busca comprender cómo se organizan los contenidos, nos permite acceder a una descripción más completa del proceso de constitución de los objetos y de la subjetividad en sí misma.

Lo que sostuvimos hasta aquí nos lleva a afirmar que la síntesis asociativa presupone la síntesis temporal. Con todo, en ocasiones, las palabras de Husserl parecen apoyar la hipótesis de que ambas poseen igual status:

Trascendentales en sentido general, como apodícticamente necesarias para la génesis de una subjetividad (la que sólo es pensable en la génesis), son también otros modos de síntesis [...] que [...] transcurren a una con la síntesis que constituye la forma temporal de todos los objetos, y así deben concernir a la vez al contenido temporal, al contenido objetivo formado temporalmente. ${ }^{14}$

Esta idea de que las síntesis de contenido operan «a una con la síntesis temporal» puede llevarnos a apoyar la tesis recién mencionada. No obstante esto, vemos en el mismo parráfo cómo la constitución temporal del material hylético precede a la conformación de las unidades hyléticas en relación propiamente con su contenido.

Otro argumento en favor de la anterior lectura aparece en relación con el estudio de la protoasociación. En este caso, la posibilidad de dejar de lado los horizontes temporales y llevar a cabo un análisis limitado al presente parece

12 Ibid., 128

13 Bégout, B. op. cit., 101

14 Husserl, 1966, 125 
apoyar la idea de que, en realidad, la asociación es fundante respecto al tiempo. Sin embargo, es necesario considerar respecto a esto que otros procesos sintéticos temporales están presupuestos, aun en el presente: «el presente concreto y pleno es una unidad a partir de una síntesis que lo abarca». ${ }^{15}$ En este sentido, Husserl afirma que junto con la estructura retención-protoimpresión-protención de la conciencia temporal, se dan otras dos síntesis. Dos operaciones que son decisivas para efectuar una indagación acerca de la protoasociación, dado que establecen de manera directa las condiciones de posibilidad de tal proceso. Se refiere, así, a las síntesis de coexistencia y sucesión. Mientras que la primera determina el modo en que todos los objetos pueden confluir en un sólo tiempo, la segunda es una síntesis de enlace que ordena unas tras otras las singularidades. Coexistencia y sucesión son, entonces, condiciones de posibilidad para la conformación de un presente concreto.

Ahora bien, según Bégout, en el análisis de la protoasociación Husserl pone la conciencia temporal entre paréntesis. ${ }^{16}$ Esta posición, que desconoce evidentemente afirmaciones husserlianas como las que hemos citado más arriba -donde, por ejemplo, se destacaba la conciencia temporal como «comienzo primordial»- se sorprende, a la vez, de que el propio Husserl se contradiga a sí mismo e intenta, por ello, justificarlo. En este sentido, se pregunta: «¿cómo dejar a un lado en la investigación de la vida pasiva originaria la conciencia temporal que constituye, aparentemente, su centro? $\rangle^{17}$ Por un lado, responde a esta pregunta centrándose en el carácter formal de la síntesis del tiempo. La abstracción efectuada por el análisis de la temporalización redundaría, en definitiva, en una desnaturalización del proceso de objetivación. Por otro lado, aborda el caso de las síntesis de la coexistencia y de la sucesión y afirma que éstas «asocian, en realidad, unidades ya constituidas, configuraciones ya establecidas, al menos primitivamente». ${ }^{18}$

En términos generales, consideramos que en ambas partes de la justificación de Bégout se confunde el orden de la fundamentación con el orden de la experiencia o, en palabras de Husserl, «lo primero en sí y lo primero para nosotros». ${ }^{19}$ Mientras que las unidades hyléticas aparecen como lo primero para nosotros, los procesos que posibilitan la conformación de dichas unidades se encuentran en un nivel de fundamentación prioritario. Si bien es cierto que no hay experiencia sin afección, también lo es que esto supone la apertura de la conciencia intencional. En este sentido, la protoimpresión como ámbito de

15 Ibid. 126

16 Ver Bégout, B., op. cit., 101-104

17 Ibid., 102

18 Ibid., 102

19 Husserl 1966, 120

Contrastes vol. XXI-N² (2016) 
donación, aun restringiendo las conexiones con las otras fases de la conciencia, implica una intencionalidad abierta a lo nuevo. Por su parte, las síntesis de la coexistencia y de la sucesión son condición de posibilidad de la asociación, dado que determinan el marco en el cual la protoasociación se efectúa. La coexistencia es coexistencia de unidades sensibles, en la medida en que la entendamos desde la experiencia. Desde un punto de vista ontológico, sin embargo, la coexistencia presenta el campo, en el cual se configuran los distintos campos sensibles (visual, táctil, auditivo, etc. ). Luego, dentro de cada campo, las unidades se destacan unas de otras y se conforman, a una con ello, propiamente en unidades. Entonces, en tanto que cada campo sensible, heterogéneo respecto a los otros campos, está integrado en función del contenido, todos los campos sensibles están unidos por la temporalidad del presente viviente; ${ }^{20}$ i. e. el tiempo determina la forma de campo que reúne ${ }^{21}$ a todos los campos sensibles. Estoy en la calle y, por ejemplo, escucho el ruido de una violenta frenada. Vuelvo la mirada y veo a dos hombres bajarse de sus respectivos autos y discutir. La reunión de la información sonora con la visual me permite colegir que un accidente estuvo a punto de ocurrir. Si no hubiese escuchado el ruido o no viera a los dos hombres, mi experiencia sería incompleta. El hecho de que sea posible saber qué fue lo que ocurrió permite notar la concurrencia de los distintos campos sensibles dentro de un marco de coexistencia que los reúne.

Por su parte, la síntesis de sucesión marca las primeras ordenaciones dentro del campo hylético, de carácter temporal, a partir de las cuales operarán sí procesos de asociación de contenido. En efecto: la concreción sólo es posible como fusión de la forma de ordenación, esto es, ordenado temporalmente. En este sentido, la continuidad interna de los datos fundamenta la fusión de contenidos. Y si buscamos, nuevamente, un ejemplo urbano de esto, podríamos pensar en el caso de un colectivo que se acerca en la noche. En la noche relativamente oscura solo puedo distinguir, primero, una luz indiferenciada. Luego, noto que son dos luces separadas a una distancia tal que me permite inferir que se trata de un vehículo de gran tamaño. A medida que se acerca, las luces se hacen cada vez más intensas, hasta el punto de que me encandilan cuando llega a la parada. El orden en la aparición de estas luces es fundamental en la constitución de la experiencia. ¿Cómo podría hablar de la experiencia del colectivo que viene si, en lugar de unas luces que se van distinguiendo y haciendo cada vez más intensas, tuviera las mismas luces pero dispuestas en orden diferente? Evidentemente, la sucesión tampoco está subordinada a los campos sensibles, pero sí a la inversa. En suma, si las síntesis temporales de coexistencia y sucesión no dependen, como creemos haber mostrado, de los 
procesos que operan en los distintos campos sensibles, sino que, por el contrario, los fenómenos temporales están en ellos presupuestos, debe aceptarse el carácter primordial de la síntesis del tiempo respecto a la asociación.

\section{LA PROTOASOCIACIÓN}

Para dar cuenta de la primordialidad del tiempo nos hemos referido ya a ciertas condiciones de la asociación. En particular, a los protofenómenos que se manifiestan en el marco de la restricción al presente viviente. Este nuevo aspecto de la reducción fenomenológica limita la investigación a lo dado en el presente, dejando de lado los horizontes de pasado y futuro. Hay que tener en cuenta, sin embargo, que no está pensando Husserl aquí en un presente puntual, como lo hará en los Manuscritos de los años ' 30 , sino en un presente ampliado que incluye la retención viviente; ${ }^{22}$ esto es: un presente en el cual se establecen las condiciones para la percepción de un objeto, pero sin intervención del predelineamiento que posibilita la experiencia pasada. Sabemos que la fenomenología genética permite describir las conexiones que hacen que cada percepción no sea una experiencia completamente nueva, sino que pueda ser anticipada en función de lo ya vivido. La reducción al presente viviente no considera estas conexiones, sólo pretende determinar las condiciones de posibilidad de la percepción en esta dimensión restringida de la conciencia.

En este marco, decíamos, es posible observar que existen unidades destacadas por sí. Estas unidades remiten a protosíntesis, como mecanismos elementales de constitución. En cuanto a la constitución pasiva temporal, tenemos las nombradas síntesis temporales de la coexistencia y la sucesión. A su vez, es posible dar con protofenómenos de la asociación que operan según leyes propias. Los principios de la semejanza, el contraste y la contigüidad nombran el modo en que se efectúan todas las síntesis de asociación; es decir: no sólo las que conciernen a la protoasociación, sino también las de la asociación reproductiva e inductiva. Respecto a estas leyes es interesante el señalamiento de Holenstein. Destaca que el recurso a estos clásicos principios de la asociación no debe tomarse como algo meramente convencional, sino como una versión propiamente fenomenológica. ${ }^{23}$ La originalidad de esta interpretación radica en la aplicación de estas leyes a una conciencia que es intencional y horizóntica. En especial, el carácter horizóntico de la conciencia marca nuevas posibilidades para la asociación, en la medida en que da cuenta de las síntesis como relaciones que se establecen entre lo explícito y lo implícito. Todo se da en la inmanencia de la conciencia, pensada ésta, a su vez, como un todo.

22 Ibid., 137-138

23 Holenstein, E. op. cit., 40 
La semejanza es el principio fundamental, a través del cual se producen enlaces de homogeneidad y heterogeneidad. Estos enlaces pueden tener mayor o menor intensidad, dependiendo del grado de semejanza que se determina entre los datos. A su vez, pueden llevarse a cabo no sólo fusiones de cercanía, sino también de lejanía. En el espacio, pero también en el tiempo no sólo se asocia lo que está próximo, se puede conectar además lo lejano. El contraste, por su parte, muestra cómo las unidades se constituyen. En el presente viviente observamos unidades sensibles que se destacan en sí. Este destacarse es, justamente, resultado del contraste que se establece entre los distintos datos hyléticos. Lo cual conduce, a la hora de analizar el fenómeno del destacarse en todas sus dimensiones, a la fenomenología de la afección. Por último, como señala Holenstein, la contigüidad juega en los Análisis un rol secundario frente a los otros dos principios. ${ }^{24}$ Permite, sin embargo, llevar a cabo enlaces en función de referencias vacías, tanto espaciales como temporales. Ahora bien, mientras que desde un punto de vista estático la semejanza da cuenta del destacarse de las unidades sensibles, desde un punto de vista genético posibilita la conciencia de identidad. En este último caso operan otros dos procesos que complementan las fusiones que posibilita la semejanza: el sobrepujamiento, que instala la comparación entre las unidades para identificar la coincidencia, y el recubrimiento, que posibilita la identificación de las unidades y la repetición de lo mismo.

Los protofenómenos de la asociación describen el modo a través del cual se constituyen las unidades sensibles preobjetivas. Estas unidades se conforman en relación con un campo sensible determinado. Esto quiere decir que los datos de color, por ejemplo, se integran en un campo visual. Para comprender con profundidad esta cuestión hay que considerar con más detalle las nociones de dato y campo. Por una parte, se podría pensar la existencia de un campo que «se va llenando» de datos sensibles; como si los datos fueran entrando en el campo de la conciencia. Pero no es en esto en lo que está pensando Husserl. Esta idea presupondría unos datos que existen de manera independiente respecto a la conciencia, cuando, de hecho, lo que se está intentando explicar es el modo en que los datos se constituyen en la inmanencia de la conciencia. Debemos tener en cuenta aquí, nuevamente, las críticas dirigidas al realismo psicologista. A su vez, la noción de conciencia como un recipiente es superada por la correlación intencional, tesis central de la fenomenología husserliana.

En relación con el concepto de dato también podríamos equivocarnos, si consideramos a los datos inmanentes como unidades aisladas. La fenomenología de la asociación no supone un atomismo, en la medida en que hay una relación intrínseca entre el campo y los datos. Así como no hay datos que no 
se den en un campo, no hay tampoco un campo sin datos. La unidad del dato que se destaca se configura en función de todo el campo, es decir que todos los otros datos no destacados están implícitos. Resulta imposible, por tanto, tomar un dato como un hecho aislado, como una unidad de sentido independiente, puesto que, en su origen, presupone la totalidad de datos correspondiente a su campo sensible.

La relación entre el campo y las unidades apunta al destacarse como un fenómeno central. Fundado en el principio del contraste, por el cual las unidades se oponen unas a otras, este fenómeno da cuenta de la conformación de las unidades sensibles como emergentes de un campo que las integra. La forma que cobra el campo responde, a su vez, a la lucha entre las sensaciones por emerger, por destacarse. En este sentido, nos hemos referido a un «en sí»» del destacarse. Sin embargo, el mismo fenómeno puede ser abordado desde un punto de vista complementario. Cada unidad que busca ser prominente, procura a la vez captar la atención del yo. De este modo, es posible considerar el destacarse como un «para». En este marco, se plantea una fenomenología de la afección, a fin de indagar las condiciones implicadas en que el sujeto se vuelva sobre lo sensible.

\section{LA AFECCIÓN}

La afección es «el estímulo conciencial, la peculiar atracción que un objeto consciente ejerce sobre el yo», ${ }^{25}$ entendiendo en este caso por «objeto» no solo los objetos constituidos perceptivamente, sino también las unidades sensibles preobjetivas, las sensaciones. Ahora bien, aunque todas las unidades emiten rayos de fuerza afectante que procuran el volverse del yo, no todas lo alcanzan efectivamente. En este sentido, Micali señala tres niveles de la afección. ${ }^{26} \mathrm{El}$ primero, que da cuenta de la tendencia a la afección que poseen las sensaciones, independientemente de toda intervención yoica. El segundo, donde el yo se ve afectado por las sensaciones, pero en un grado bajo. El tercero y último, en el que la atención yoica es despertada afectivamente. Si bien puede establecerse esta distinción en niveles, hay que tener en cuenta que todo el proceso afectante tiene un carácter gradual que depende de la intensidad de las sensaciones, dado que ésta marca la fuerza afectante capaz de motivar la atención yoica. Tenemos, así, una gradualidad de la afección que es correlativa de la gradualidad del contraste que opera entre las unidades sensibles. Ambas gradualidades pueden vincularse a partir del destacarse; i. e.: más alto el contraste, más se destaca una unidad, más capacidad de afectar posee. Mientras que unas unidades pueden permanecer para siempre en el trasfondo de la vida activa, hay otras

25 Husserl 1966, 148.

26 Micali 2008, 69.

Contrastes vol. XXI-N² (2016) 
que pueden despertar fácilmente el volverse del sujeto. Con todo, es necesario insistir en que, aunque una unidad nunca pase al primer plano de la conciencia, no significa que no opere en la actividad. La tendencia a la afección que se le asigna per se a toda unidad sensible habla de que aun en un grado ínfimo todo puede afectar ${ }^{27}$ o, que, al menos, «lo restante estimula». ${ }^{28}$ En efecto, el hecho de que algo llegue a ser activamente consciente está motivado por múltiples factores que exceden el estímulo afectante: sentimientos sensibles y preferencias instintivas condicionan en última instancia el volverse-hacia efectivo. ${ }^{29}$

En correlación con los niveles de la afección, pueden reconocerse en el presente viviente distintos tipos de configuración de los campos sensibles. Nos referimos al relieve como la forma que adquiere el campo en función de las unidades que se destacan. La figura sirve para mostrar la relación intrínseca entre el campo y las unidades, en la medida en que las unidades se conforman a partir del campo al cual pertenecen. Así como los picos y los valles no son nada sin la montaña, las unidades sensibles emergen de los campos. Si tomamos el destacarse desde un punto de vista genético, podemos ver que los campos sensibles modifican permanentemente su forma, a la vez que las unidades van perdiendo y ganando entre sí fuerza afectante. En función de la atracción que las unidades ejercen sobre el yo se determina una triple estratificación del relieve. En primer lugar, un relieve de notoriedad (Merklichkeitsrelief) que coincide con el nivel ínfimo de fuerza. En segundo lugar, un relieve de perceptibilidad (Bemerksamkeit), en relación con el volverse primero del yo. En tercer lugar, un relieve de la atención (Aufmerksamkeit), que muestra la conformación que alcanza la atención yoica. También en este caso el proceso de modificación de los campos implica una gradualidad que tiene que ver con la vivacidad que van logrando las distintas unidades y correlativamente la consciencia de éstas que adquiere el sujeto.

Junto con el nivel de fuerza afectante que posee una unidad sensible como resultado del contraste, operan otros mecanismos que posibilitan también el destacarse. Husserl se refiere, así, a las evocaciones asociativas: «Dentro de cada presente viviente, y limitados ante todo a los datos sensibles que se unifican en él, actúan constantemente más allá de sí las afecciones, encontramos continuamente evocaciones asociativas». ${ }^{30}$ La evocación es el proceso que permite describir cómo se propaga la fuerza afectante desde el presente impresional hacia toda la cadena retencional. En este sentido, representa un proceso que

27 Husserl 1966, 161.

28 Ibid., 150.

29 Este tema de las motivaciones instintivas y sentimentales es tematizado nuevamente por Husserl en los Manuscritos del grupo C (Späte Texte über Zeitkonstitution (1929-1934). Die C-Manuskripte. Husserliana Materialien VIII). Ver, por ejemplo, el escrito C 10.

30 Husserl 1966, 157-158 
vincula el tiempo y la asociación, en la medida en que articula lo que ocurre en el relieve conformado en el presente viviente con todo el resto de la conciencia.

\section{LA ASOCIACIÓN Y LA RELACIÓN PRESENTE-PASADO}

Hasta este punto nos hemos referido meramente a la organización del campo sensible en el presente. A partir del presente como fuente originaria de toda afección se produce un proceso de conservación y propagación de la fuerza afectante. La modificación retencional es reinterpretada, ahora, en términos afectantes. El desvanecimiento que entraña el alejarse del ahora impresional es considerado, luego, como una pérdida gradual de fuerza afectante. Por un lado, tenemos un proceso de pérdida de intuitividad que se corresponde con el presente extendido de la percepción. Es decir, mientras las retenciones auxilian a la protoimpresión en la conservación de los aspectos recién sidos de un objeto, se produce en ellas una pérdida gradual de intuitividad. Cuando el objeto sale del tema, las retenciones pierden totalmente la intuitividad. Nos referimos a las retenciones que participan de la intuividad como retenciones cercanas. Con todo, el proceso retencional continúa más allá del horizonte intuitivo. Las retenciones lejanas son las que conservan todo lo que hemos experienciado, desplazándose cada vez más hacia el pasado. En este caso, se habla de una pérdida de fuerza afectante. Dice Husserl: «Con el llegar a ser nulo de la intuitividad no es, pues, nula la fuerza afectante». ${ }^{31}$

Se vislumbra en este proceso de pérdida de afección el final como grado de cero de la fuerza. Damos, entonces, con la oscuridad total del inconsciente: «irresistiblemente se pierde el resto de la distinción y de la fuerza afectante que se había derivado retencionalmente del punto originario».$^{32} \mathrm{El}$ inconsciente es entendido, así, como la plena indiferenciación, la carencia total de fuerza. Resulta necesario señalar aquí la ambigüedad de la posición husserliana frente a la cuestión. Por un lado, las referencias recién aludidas en torno al grado de cero de la afección. Por otro lado, la tendencia a la afección como un grado ínfimo que debe poseer cada unidad sensible. El problema que se le presenta a Husserl es que, si no le asigna a cada unidad dicha tendencia a la afección, no se explica cómo algo que no afecta en absoluto pueda llegar a afectar. ${ }^{33}$ Junto con esto, aparece otro principio que dice que siempre podemos volver a hacer intuitivo aquello ya vivido. ${ }^{34}$ Luego, es necesario que el vacío indiferenciado en que deviene el inconsciente no sea equiparado a una carencia total de la fuerza. El enigma, con todo, queda abierto. 
Ahora bien, la noción de evocación conecta la fenomenología de la afección en el presente viviente con la asociación reproductiva e inductiva. Los principios que operan aquí son las mismas leyes asociacionistas (semejanza, contraste y sucesión), aunque no limitados en este caso a la esfera del presente, sino incorporados al análisis de toda la vida de la conciencia. Recordemos que la asociación reproductiva aparece como la primera motivación para llevar a cabo una fenomenología de la asociación. En particular, se destaca el nuevo modo de referirse a la conciencia intencional, en términos de conciencia evocadora. En este sentido, se afirma: «lo presente recuerda lo pasado». ${ }^{35}$ Así, describir la relación entre lo explícito y lo implícito permite entender en qué medida la experiencia pasada interviene en el presente. Tanto a la hora de conformar las objetividades en función de tipos adquiridos, como en la proyección del futuro basado en lo ya vivido. A su vez, esta dirección de la investigación posibilita consolidar una concepción más completa del sujeto, puesto que descubre las motivaciones de la rememoración en la vida yoica. ${ }^{36}$ La asociación reproductiva como una teoría de las rememoraciones efectivas y posibles ofrece los recursos para resolver varias cuestiones en torno a la rememoración. En el texto husserliano aparecen los problemas de la fusión de los recuerdos y los recuerdos falsos. También se brindan soluciones a la hora de entender qué ocurre con otros fenómenos complejos de la rememoración; entre ellos, el recuerdo de la primera experiencia y los recuerdos de la temprana infancia. Por todo esto, la asociación reproductiva es considerada por Husserl como «la teoría de la asociación en su sentido más primario y más propio». ${ }^{37}$

Pueden señalarse cuatro características que posee la fenomenología de la asociación a este nivel:

1. Es un análisis noemático. «No decimos que vemos en todas partes el recordar algo», ${ }^{38}$ sino que se estudia el correlato objetivo, los recuerdos.

2. Es un estudio de la conciencia de horizonte. Dado que se indaga la relación entre lo destacado y lo implícito, se asume que lo singular presupone lo plural.

3. Es un abordaje de la relación de semejanza y cumplimiento en términos genéticos. Esto se evidencia en la relación entre lo evocante y lo evocado como formando parte de un proceso. 
4. Tiene en cuenta tanto asociaciones mediatas como inmediatas. Cuando estas últimas pasan inadvertidas accedemos a ellas por medio de la reflexión.

Mientras que la asociación reproductiva da cuenta de la relación presentepasado, la asociación inductiva muestra el vínculo entre la fase actual y el futuro. En la medida en que supone la constitución del presente y las evocaciones entre el pasado y el presente, la génesis de las esperas se encuentra en un grado de fundamentación superior. ${ }^{39}$ Afirma Husserl que «lo semejante recuerda lo semejante pero también permite esperar lo semejante». ${ }^{40}$ En este sentido, esta operación da cuenta del modo según el cual lo ya vivido permite anticipar lo por venir. Interviene para esto una causalidad motivacional que hay que distinguir de la causalidad psicofísca, en la medida en que sólo establece relaciones posibles entre el pasado y el futuro y no causalidades determinísticas. Como ya indicamos la proyección del futuro se lleva a cabo por medio de un proceso inductivo. Esto resulta en una gradualidad o probabilidad de la proyección en función de la frecuencia del haber aparecido. Si bien no existe una explicación pormenorizada de cómo esto sucede, es posible afirmar que la asociación inductiva da cuenta de la conformación de los hábitos y sus correlatos, los tipos empíricos. A grandes rasgos, esto tiene que ver con las posibilidades de predelineamiento de la experiencia futura en base a la pasada que posibilita la evocación.

\section{REFERENCIAS BIBLIOGRÁFICAS}

BÉGOUT, B. 2000: La généalogie de la logique. Paris: Librairie Philosophique.

BERNET, R. 1982: «Is the Present Ever Present? Phenomenology and Metaphysics of Presence» en Research in Phenomenology, Vol. XII.

BERNET, R. 1983: «La Présence du Passée dans l'Analyse Husserlienne de la Conscience du Temps». Revue de Métaphysique et Morale. Vol. 88: Nro. 2.

BROUGH, J. B. 1993: «Husserl and the deconstruction of time» en The Review of Metafisics. Vol. 46: №3.

BROUGH, J. 1977: «The Emergence of an Absolute Counsciusness in Husserl's Early Writings on Time-Consciousness» en ELLISTON, F. A. y Mc CORMICK, P. 1977: Husserl. Expositions and Appraisals. Notre Dame/Londres: University of Notre Dame Press.

DERRIDA, J. 1995: La voz y el fenómeno. Valencia: Pre-textos. 
FERRER SANTOS, U. 2008: La trayectoria fenomenológica de Husserl. Pamplona: Eunsa.

GARCÍA-BARÓ, M. 1998: «Introducción a los problemas que afectan a la noción fenomenológica de mundo de la vida» en PINTO, Ma. L y GONZÁLEZ LÓPEZ, J. L. 1998: Fenomenología y ciencias humanas. Santiago de compostela: Universidad de Santiago de Compostela.

HELD, K. 1966: Lebendige Gegenwart. The Hague: Martinus Nijhoff.

HOLENSTEIN, E. 1972: Phänomenologie der Assoziation. Zu Struktur und Funktion eines Grundprinzips der passiven Genesis bei E. Husserl. La Haya: Martinus Nijhoff.

HUSSERL, E. 1966: Analysen zur passiven Synthesis. Husserliana XI. La Haya: Martinus Nijhoff.

HUSSERL, E. 1973: Cartesianische Meditationen und pariser Vorträge. Husserliana I. La Haya: Martinus Nijhoff.

HUSSERL, E.: 1996: Meditaciones Cartesianas. México: Fondo de Cultura Económica.

HUSSERL, E. 2006: Späte Texte über Zeitkonstitution (1929-1934). Die C-Manuskripte. Husserliana Materialien VIII. Dordrecht: Springer.

HUSSERL, E. 1969: Zur Phänomenologie des inneren Zeitbewusstesens (1893-1917). Husserliana $X$. Tübingen: Max Niemayer.

MICALI, S. 2008: Die Überschüsse der Erfahrung. Grenzdimensionen des Ich nach Husserl. Dordrecht: Springer.

Verónica Kretschel es Doctora en Filosofía (Universidad de Buenos Aires); Becaria posdoctoral Conicet; Docente en Filosofía Contemporánea (FFyL-UBA) y Filosofía (FCsSs-UNLZ).

Líneas de investigación:

Flosofía contemporánea, Fenomenología y Fenomenología del tiempo.

\section{Publicaciones:}

Kretschel, V., «Tiempo y asociación. Acerca de la relación entre los Manuscritos de Bernau y los Análisis sobre la síntesis pasiva», Investigaciones Fenomenológicas, Nro. 11, 2014, 143-163 (issn 1885-1088)

Kretschel, V., «Husserl y la metafísica: la relación protoimpresión-retención», Anuario Filosófico, Vol. 46., Nro. 3, 2013, 543-563

Correo electrónico: veronicakretschel@gmail.com 
